\title{
Engineered receptor binding domain immunogens elicit pan-sarbecovirus neutralizing antibodies outside the receptor binding motif
}

Blake M. Hauser ${ }^{1 \#}$, Maya Sangesland ${ }^{1 \#}$, Evan C. Lam ${ }^{1}$, Kerri J. St. Denis ${ }^{1}$, Jared Feldman ${ }^{1}$, Ashraf S. Yousif ${ }^{1}$, Timothy M. Caradonna ${ }^{1}$, Ty Kannegieter ${ }^{1}$, Alejandro B. Balazs ${ }^{1}$, Daniel Lingwood $^{1 *}$ and Aaron G. Schmidt ${ }^{1,2^{*}}$

\section{Affiliations:}

${ }^{1}$ Ragon Institute of MGH, MIT and Harvard, Cambridge, MA, 02139, USA

11 (dlingwood@mgh.harvard.edu)

12 \# These authors contributed equally. 
Abstract: Effective countermeasures are needed against emerging coronaviruses of pandemic potential, similar to severe acute respiratory syndrome coronavirus 2 (SARS-CoV-2). Designing immunogens that elicit broadly neutralizing antibodies to conserved viral epitopes on the major surface glycoprotein, spike, such as the receptor binding domain (RBD) is one potential approach. Here, we report the generation of homotrimeric RBD immunogens from different sarbecoviruses using a stabilized, immune-silent trimerization tag. In mice, we find that a cocktail of these homotrimeric sarbecovirus RBDs elicits antibodies to conserved viral epitopes outside of the ACE2 receptor binding motif $(\mathrm{RBM})$. Importantly, these responses neutralize all sarbecovirus components even in context of prior SARS-CoV-2 imprinting. We further show that a substantial

Author summary: Immunity to SARS-CoV-2 in the human population will be widespread due to natural infection and vaccination. However, another novel coronavirus will likely emerge in the future and may cause a subsequent pandemic. Humoral responses induced by SARS-CoV-2 infection and vaccination provide limited protection against even closely related coronaviruses. We show immunization with a cocktail of trimeric coronavirus receptor binding domains induces a neutralizing antibody response that is broadened to related coronaviruses with pandemic potential. Importantly, this broadening occurs in context of an initial imprinted SARS-CoV-2 spike immunization showing that preexisting immunity can be expanded to recognize other related 
37 coronaviruses. Our immunogens focused the serum antibody response to conserved epitopes on

38 the receptor binding domain outside of the ACE2 receptor binding motif; this contrasts with

39 current SARS-CoV-2 therapeutic antibodies, which predominantly target the receptor binding

$40 \quad$ motif. 


\section{$41 \quad$ Main Text:}

\section{Introduction}

43 The emergence of the novel severe acute respiratory syndrome coronavirus 2 (SARS-CoV-2) and

44 the subsequent global pandemic has highlighted the disruptive threat posed by viruses for which

45 humans have no prior immunity. Rapid development of potential vaccines has led to an

46 unprecedented 40 candidates already in Phase 3 clinical trials or approved since January 2020;

47 while differing in modality (e.g., mRNA, adenovirus), the primary immunogen for many of these

48 candidates is the SARS-CoV-2 spike ectodomain (1). With the continued global spread of SARS-

$49 \mathrm{CoV}-2$, in conjunction with potential vaccinations, it is likely that a large proportion of the global

50 population will eventually develop an immune response to SARS-CoV-2. However, even after

51 potentially achieving herd immunity sufficient to slow the spread of SARS-CoV-2, there remains

52 a constant threat of emerging coronaviruses with pandemic potential. Indeed, surveillance efforts

53 have identified numerous unique coronaviruses within various animal reservoirs, raising the

54 possibility of zoonotic transmission $(2,3)$. Such events are likely to increase in frequency as a

55 result of human impact on the environment (4). While the current SARS-CoV-2 pandemic has an

56 estimated infection fatality rate of between $\sim 1-2 \%$, with numerous cases likely undetected,

57 previous SARS-CoV and MERS-CoV outbreaks were more lethal with $\sim 10 \%$ and $\sim 35 \%$ case

58 fatality rates, respectively, raising the possibility that future novel coronaviruses will potentially

59 have high mortality (5-7). Additionally, elicited immunity to SARS-CoV-2 infection may not

60 protect against even closely related novel coronaviruses from the same sarbecovirus subgenus $(8$,

619 9). It is therefore critical to not only address the current pandemic, but also develop vaccine

62 platforms that can be readily adapted to potential emerging coronaviruses. 
64 While we cannot readily predict which coronaviruses will next emerge into the human population,

65 a proactive approach to generate broadly protective immunity is to design immunogens that elicit

66 humoral responses targeting conserved sites on the coronavirus spike glycoprotein. Such sites may

67 include the receptor binding domain which engages host-cell receptors necessary to facilitate viral

68 cell entry; these spike-mediated interactions are conserved across coronaviruses (10). Indeed, a

69 subset of spike-directed antibodies from convalescent patients can potently neutralize SARS-CoV-

70 2; comparable neutralizing antibodies against SARS-CoV and MERS-CoV have also been

71 identified $(6,11-16)$. Some spike-directed antibodies that neutralize SARS-CoV-2 also bind the

72 SARS-CoV spike protein, highlighting the presence of cross-reactive neutralizing epitopes (11,

73 17, 18). Multimerized versions of the receptor binding domains (RBDs) of several coronaviruses

74 have previously been shown to be potent immunogens (19). Here, we describe a customizable

75 vaccine that elicits pan-sarbecovirus neutralization against both SARS-CoV-2 and the potentially

76 emergent WIV1-CoV (20). This approach focuses antibody responses to conserved, protective

77 RBD epitopes shared across sarbecoviruses. Its flexible nature allows facile interchanging of

78 potential vaccine strains updated to confer neutralization against new emerging coronaviruses.

79 Responses focused to these conserved epitopes maintain potent SARS-CoV-2 neutralization

80 activity despite minimal ACE2 receptor binding motif coverage. Importantly, this approach was

81 applied in context of "pre-existing" SARS-CoV-2 humoral immunity with the goal of broadening

82 neutralization while simultaneously boosting the neutralizing antibody response to SARS-CoV-2,

83 similar to the "back boost" effect of seasonal influenza immunizations (21). Thus, it has the

84 potential to provide protection against currently circulating SARS-CoV-2, while proactively

85 generating neutralizing antibody responses against emerging coronaviruses. 


\section{$87 \quad$ Results}

Designing a Trimerized Receptor Binding Domain Construct Using a Non-Immunogenic Tag

We designed a cystine-stabilized and hyperglycosylated variant of a GCN4 trimerization tag to generate a homotrimeric immunogen "cassette" to rapidly exchange RBDs from various hyperglycosylated GCN4 allows the RBDs to remain trimerized while the tag is "immune silent" via immobilized metal affinity chromatography followed by size exclusion chromatography; the trimeric species was confirmed using SDS-PAGE analysis under non-reducing conditions (Fig.

1B-C). Their antigenicity was assayed using conformational-specific antibodies CR3022 and/or to RBD monomers (Fig. S1). The 8xHis purification tags were removed by HRV 3C protease enzymatic cleavage prior to immunization.

\section{Immunization Regimens Generate Cross-Reactive Antibody Responses}

To understand how preexisting immunity to SARS-CoV-2, whether acquired through natural infection or vaccination, could affect immune responses to our immunogens, we primed our cohorts with recombinant spike or $\operatorname{RBD}$ protein $(23,24)$. Our two control arms followed this prime with subsequent homologous boosts of recombinant SARS-CoV-2 spike (“Spike” cohort) or RBD ("RBD" cohort); the latter is necessary for comparing the effect on immunogencity of monomeric 
110 versus trimeric RBDs. Our experimental arms both received the spike prime and included a SARS-

111 CoV-2 RBD homotrimer boost ("Homotrimer" cohort) and an equimolar boosting cocktail of 112 SARS-CoV-2, SARS-CoV, and WIV1-CoV RBD homotrimers (“Cocktail” cohort) (Fig. 2A). All 113 cohorts received the same total amount of protein in each immunization, $20 \mu \mathrm{g}$, adjuvanted with 114 Sigma Adjuvant (25). Immunizations were performed at days 0, 21, and 42. We evaluated the 115 serum response against coronavirus-derived antigens using ELISAs, including SARS-CoV-2 116 spike, RBDs from SARS-CoV-2, SARS-CoV, WIV1, as well as a SARS-CoV-2 $\triangle$ RBM RBD with 117 two engineered glycans that abrogate ACE2 engagement (Fig. 2B, S2A, S3A-C). All four 118 immunization regimens resulted in similar patterns of serum reactivity. Each cohort demonstrated 119 a significant decrease in reactivity against SARS-CoV RBD as compared to the SARS-CoV-2 120 RBD or spike, though the magnitude of this difference was relatively small. The cohort which 121 received the RBD homotrimer cocktail boost had the highest overall endpoint titers, while the 122 cohort that received the monomeric SARS-CoV-2 RBD had the lowest overall endpoint titers; the 123 latter observation is consistent with other previous reports and likely due to the inefficiency of 124 monomeric RBDs effectively stimulating B cell receptors. We confirmed the non-immunogenic 125 nature of the hyperglycosylated GCN4 tag by assaying sera from the wildtype homotrimer cocktail 126 boost against another viral glycoprotein, hemagglutinin, with the same tag (Fig. S2B).

Immunization with a Cocktail of Homotrimeric Receptor Binding Domains Focuses the Immune 129 Response to Cross-Reactive Epitopes

130 We next evaluated whether the serum response was directed towards cross-reactive, and 131 potentially broadly neutralizing RBD epitopes. Conservation across SARS-CoV-2, SARS-CoV, 132 and WIV1-CoV RBDs primarily occurs outside of the ACE2 receptor binding motif (RBM). 
Indeed, the previously characterized CR3022 and S309 antibodies have footprints that together cover much of this conserved region, with epitope buried surface area (BSA) of $917 \AA^{2}$ and 795 $\AA^{2}$ respectively in comparison to BSA of $869 \AA^{2}$ for $\operatorname{ACE} 2(17,18,26)$. We performed serum competition by incubating RBD-coated ELISA plates with IgGs B38, P2B-2F6, CR3022, and S309, representing each of the four previously defined "classes" of SARS-CoV-2 RBD epitopes (27) (Fig. S2C). We then assessed binding of mouse serum IgG. In all cohorts, competition with both CR3022 and S309 significantly reduced serum titers against the SARS-CoV and WIV1-CoV

RBDs (Fig. 2C, S2C). However, only the cohort receiving the RBD homotrimer cocktail showed a significant reduction in serum titers against the SARS-CoV-2 RBD in competition with both CR3022 and S309 ( $\mathrm{p}=0.0340)$ (Fig. 2C). This result suggests a higher degree of focusing to this

To compare the observed sera responses, we measured the amount of antigen-specific IgG B cells expanded by the Spike, Homotrimer, and Cocktail immunization regimens. Low antigen-specific

ELISA titers for the RBD cohort indicated that we were unlikely to be able to robustly quantitate antigen-specific B cells, therefore mice from that cohort were excluded from subsequent analyses. We engineered a SARS-CoV-2 RBD variant that has two additional glycans on the RBM which effectively block ACE2 engagement (Fig. S3A-C). This variant allowed us to bin SARS-CoV-2 spike-directed B cells into 3 populations: those that bound RBM epitopes; those that bound the non-RBM epitopes on the RBD; and those that bound the "remainder" of the spike protein (Fig. S3D-E). As a subset of SARS-CoV-2 spike-directed B cells, the proportion of B cells specific for the SARS-CoV-2 RBM and non-RBM portion of the RBD were considerably higher in the cohorts 
receiving the SARS-CoV-2 RBD homotrimer and RBD homotrimer cocktail boosts $(\mathrm{p}=0.0070)$

(Fig. 3A). We also binned B cells that bound to both the SARS-CoV-2 spike and either the SARS-

CoV RBD or WIV1-CoV RBD (Fig. 3B). We found that cross-reactive IgG B cells predominantly targeted epitopes outside the RBM (the spike remainder a result of the prime), mirroring what we

160 observed in sera responses.

Elicited Immune Response is Cross-Neutralizing and Targets Non-Receptor Binding Motif Epitopes

We next determined the neutralization potency from each of our cohorts using SARS-CoV-2, SARS-CoV, and WIV1-CoV pseudoviruses $(8,9)$. We obtained NT50 values when possible; we note that most serum samples for which NT50 values could not be determined still had some weak neutralizing activity (20 out of 24 samples) (Fig. S4E). We observed a significant increase in WIV1-CoV neutralization in the cohort that received the homotrimer cocktail boost compared to all other cohorts $(p=0.0012)$ (Fig. 4A). Importantly, this did not result in any significant loss in serum neutralization potency against SARS-CoV-2 $(p=0.6594)($ Fig. 4B). We also observed higher levels of SARS-CoV neutralization in the cohort that received the homotrimer cocktail boost as compared to the cohort boosted with the SARS-CoV-2 RBD homotrimer and the SARSCoV-2 monomeric RBD, though this trend was not significant $(\mathrm{p}=0.2370)$ (Fig. 4C). The samples from the cohort that received the homotrimer cocktail boost where an NT50 value could not be obtained, nevertheless still show some evidence of weak neutralization.

To assess cross-neutralization of related coronaviruses that were not included in the RBD homotrimer cocktail cohort, we performed neutralization assays using RaTG13-CoV and 
SHC014-CoV pseudoviruses, both of which are members of the sarbecovirus subgenus that have been detected in bats but not in humans (Fig. 4D) $(28,29)$. These viruses, along with WIV1, are ACE2-using sarbecoviruses in animal reservoirs that could enter the human population, similar to SARS-CoV and SARS-CoV-2. RaTG13-CoV is closely related to SARS-CoV-2 with 89.6\% amino acid identity within the RBD, while SHC014 is more distant with $76.3 \%$ identity. For RatG13-CoV, we observed similar neutralization patterns whereby the RBD cohort had significantly lower neutralization than the Spike and Cocktail cohorts. (Fig. 4D-E). For SHC014$\mathrm{CoV}$, however, the cohort that received the RBD homotrimer cocktail boosts showed significantly greater neutralization compared to all other cohorts $(p=0.0145)($ Fig. 4D, F). Furthermore, the corresponding ELISA titers also show no loss in binding to the SHC014-CoV and RaTG13-CoV RBDs relative to SARS-CoV-2 RBD (Fig. S2D-E). Thus, compared to other cohorts, immunization with the RBD homotrimer cocktail resulted in a neutralizing antibody response with both retrospective (e.g., SARS-CoV) and prospective breadth (e.g., WIV1-CoV, SHC014-CoV, and RaTG13-CoV) even in context of preexisting immunity to SARS-CoV-2.

We wanted to determine what fraction of SARS-CoV-2 neutralization could be attributed to the non-RBM RBD-directed response. To that end, we performed adsorption of pooled sera from each cohort using MERS-CoV RBD (negative control), SARS-CoV-2 RBD, and a SARS-CoV-2 RBD with four additional glycans engineered onto the RBM (SARS-CoV-2 $\triangle$ RBM RBD) (Fig. S3B, S4A-D). This construct was designed to block all RBM-directed antibodies as opposed to the one used in flow cytometry, which only block antibodies that directly compete with ACE2. Samples adsorbed with all three RBD constructs showed decreased neutralization compared to serum due to the dilution involved in the adsorption protocol. Compared to the samples adsorbed using MERS 
202 RBD, the samples adsorbed with either the SARS-CoV-2 RBD or the SARS-CoV-2 $\triangle$ RBM RBD 203 demonstrated a significant loss of SARS-CoV-2 neutralization $(p=0.0343)($ Fig. 4G, S4E). This 204 indicates that non-RBM RBD-directed antibodies alone are able to confer significant SARS-CoV2052 neutralization. We performed similar adsorption experiments on 24 post-vaccination human 206 serum samples from a previously characterized vaccine cohort (9). These patients had received 207 either 1 or 2 doses of the Pfizer (BNT162b2) or Moderna (mRNA-1273) vaccines. Similar to our 208 murine observations, human samples adsorbed using either the SARS-CoV-2 RBD or the SARS209 CoV-2 $\triangle$ RBM RBD lost significant neutralization compared to samples adsorbed using MERS 210 RBD $(p=0.0253)$ (Fig. 4H). Still, samples adsorbed with SARS-CoV-2 $\triangle$ RBM RBD did show 211 higher neutralization activity relative to samples adsorbed with SARS-CoV-2 RBD across both 212 the human and mouse samples. This indicates that RBM- and non-RBM antibodies targeting the 213 RBD play important roles in conferring SARS-CoV-2 neutralization.

\section{Discussion}

Here, we demonstrate that a cocktail of homotrimeric sarbecovirus RBDs can effectively generate a neutralizing response to all components and additional related sarbecoviruses without a bias resulting from an initial SARS-CoV-2 imprinting. We find that this cross-neutralizing antibody response is predominantly directed to RBD epitopes outside of the RBM. This contrasts SARSCoV-2 infection which does not appear to reliably generate cross-neutralizing antibodies $(8,9)$. Previous analysis into the epitopes targeted by this cross-neutralizing response has not been conducted (30). Furthermore, previously reported vaccine candidates that aim to generate such a cross-neutralizing response have not been shown to successfully focus immunity towards cross- 
neutralizing epitopes following an initial SARS-CoV-2 spike immunization, but instead were

226 tested only in a naïve homologous prime/boost format (30). However, our results suggest that 227 following an initial SARS-CoV-2 exposure (e.g., vaccination, infection), subsequent boosting with 228 a surveillance-informed selection of sarbecovirus RBD homotrimers could result in pan229 sarbecovirus immunity that protects against future pandemics despite targeting non-RBM epitopes. Whereas currently available vaccine candidates, particularly mRNA-based vaccines, appear to largely provide effective protection against currently circulating SARS-CoV-2 variants of concern, it remains unclear whether currently approved vaccines will provide protection against emerging sarbecoviruses (31-35). Immunization with SARS-CoV-2 spike-based vaccines, as well as SARS-CoV-2 infection, results in a significant loss in neutralization against existing preemergent sarbecoviruses in both humans and animal models $(8,9,36)$. SARS-CoV-2 spike-based vaccines also have reduced in vivo protection against SARS-CoV and WIV1 compared to SARSMonomeric as well as numerous SARS-CoV-2 multimerized RBD-based vaccine constructs have CoV-2 (36). been published recently and are in various stages of preclinical and clinical testing (19, 30, 37-44). However, these multimerization platforms present additional epitopes that can give rise to a scaffold-specific antibody response, which has the potential to alter hierarchies of immunodominance. Our non-immunogenic hyperglycosylated, cystine-stabilized GCN4 tag improves upon a previous hyperglycosylated version of the tag that showed markedly reduced immunogenicity (22). This may be due to the implementation of cystine-stabilization limiting the accessibility of epitopes within the coiled-coil interface of the GCN4 tag that may not be fully shielded by engineered glycans. 
This immunogen cocktail provides a framework upon which further studies to optimize a pancoronavirus vaccine can build to generate optimal broadly neutralizing antibody responses. This could include antibodies targeting the $\mathrm{N}$-terminal domain as well as the RBD, possibly elicited by vaccine regimens including full-length spike proteins. SARS-CoV-2 pseudovirus neutralization assays may underestimate the contributions of antibodies that target epitopes outside the RBD, though it remains unclear the extent to which this occurs when measuring a polyclonal serum response and against other coronaviruses (45-47). Still, most potently neutralizing monoclonal antibodies and immunization-elicited neutralizing antibodies in humans receiving the approved

While the durability of vaccine or infection-elicited antibody responses to SARS-CoV-2 remains mRNA vaccines appear to target the RBD, emphasizing the importance of shaping the RBDdirected immune response with any potential future boosting immunizations given the likely impending ubiquity of vaccine-elicited immunity (48-51). to be seen, data from seasonal coronaviruses, as well as SARS-CoV and MERS-CoV, suggests that immunity appears to wane after several years and can vary in potency between individuals (52-58). Thus, if herd immunity is not achieved or if antigenic drift of SARS-CoV-2 necessitates reformulation of current vaccines, it may present an opportunity to incorporate immunogens based on emerging coronaviruses identified through surveillance (20); importantly, based on the data presented here, such incorporation would not be at the detriment of neutralizing activity against SARS-CoV-2. Assessing in vivo protection efficacy of this vaccine regimen against SARS-CoV2 , in context of preexisting immunity to SARS-CoV-2, is hindered by the observation that a single SARS-CoV-2 spike immunization alone provides notable in vivo protection (59). 
In addition to informing future immunization regimens, these findings demonstrate that potent cross-neutralizing antibodies can target epitopes outside of the RBM on the SARS-CoV-2 RBD. Our results further demonstrate that non-RBM neutralization via the RBD forms a significant fraction of SARS-CoV-2 immunity elicited in in humans. Many potently neutralizing monoclonal antibodies currently in therapeutic development target RBM epitopes in regions of the RBD with minimal conservation across members of the sarbecovirus subgenus $(48,51)$. Consequently, these antibodies are unlikely to provide protection against future emerging coronaviruses. Identifying neutralizing antibodies targeting conserved epitopes outside the RBM and the appropriate immunizations strategies and modalities to elicit them may provide the broad protection necessary for pan-coronavirus immunity $(11,18,49)$. Furthermore, targeting these non-RBM, but still conserved epitopes, may also reduce the likelihood of antibody escape, which have already been documented for existing SARS-CoV-2 monoclonal antibodies $(45,60)$. Immunogens that facilitate immune focusing to conserved RBD epitopes, while still presenting the SARS-CoV-2 RBM, can generate a cross-neutralizing antibody response in addition to eliciting SARS-CoV-2-directed antibodies belonging to the potently neutralizing classes of RBM-directed antibodies.

While the currently approved vaccines use an mRNA platform to present SARS-CoV-2 spike, additional immunization strategies use protein-based multivalent constructs (19, 30, 37). Collectively these efforts are designed to elicit both SARS-CoV-2 and, in some cases, pancoronavirus immunity. The approach described here demonstrates a potential alternate but complementary path to generate neutralizing antibodies against multiple sarbecoviruses even in the context of prior immunity to SARS-CoV-2. Furthermore, this cocktail-based approach using 
294 trimeric immunogens parallels the current influenza vaccine composition, which aims to elicit 295 broad immunity to each constituent strain. This proof-of-principle study indicates that a similar 296 surveillance-based approach could be applied to future coronavirus vaccines, that protect against 297 future emerging coronaviruses while also maintaining protection against SARS-CoV-2. 


\section{Materials and Methods}

$302 \quad$ Receptor Binding Domain and Homotrimer Expression and Purification

303 Receptor binding domains (RBDs) were designed based on the following sequences: SARS-CoV-

3042 RBD (Genbank MN975262.1), SARS-CoV RBD (Genbank ABD72970.1), WIV1-CoV RBD

305 (Genbank AGZ48828.1), and MERS-CoV RBD (Genbank AHI48572.1). Constructs were codon

306 optimized by Integrated DNA Technologies, cloned into pVRC, and sequence confirmed by

307 Genewiz. The spike plasmid was obtained from Dr. Jason McLellan at the University of Texas, 308 Austin. It contained a Foldon trimerization domain as well as C-terminal HRV 3C-cleavable 6xHis 309 and 2xStrep II tags. Proteins were expressed in Expi293F cells (ThermoFisher) using 310 Expifectamine transfection reagents according to the manufacturer's protocols. All proteins 311 included a C-terminal HRV 3C-cleavable 8xHis tag to facilitate purification. Monomeric RBD 312 proteins also contained SBP tags, while homotrimeric constructs contained a previously published 313 hyperglycosylated GCN4 tag with two additional C-terminal cystines (22). A linker with the 314 sequence GAGSSGSG separated each RBD from the hyperglycosylated GCN4 tag. Versions of 315 the MERS-CoV RBD, SARS-CoV-2 $\triangle \mathrm{RBM} R B D$ with four additional putative glycosylation sites 316 (Figure S5), and SARS-CoV-2 RBD with C-terminal 8xHis and SNAP tags (61) were also 317 generated.

319 Transfections were harvested after 5 days and clarified via centrifugation. Cell supernatants were 320 passaged over Cobalt-TALON resin (Takara) for immobilized metal affinity chromatography via 321 the $8 x$ His tag. After elution, proteins were passed over a Superdex 200 Increase 10/300 GL (GE 322 Healthcare) size exclusion column in PBS (Corning). Prior to immunization, 8xHis tags were 323 cleaved using HRV 3C protease (ThermoScientific). Cleaved protein was repurified using Cobalt- 
324 TALON resin in order to remove the protease, cleaved tag, and any uncleaved protein. 325 Approximate purified homotrimer yields per liter of Expi293F transfected were as follows: SARS326 CoV-2 - 3 mg; SARS-CoV - 20 mg; WIV1-CoV - 25 mg.

329 Genes for the variable domains of the heavy and light chains were codon optimized by Integrated 330 DNA Technologies and cloned into pVRC constructs containing the respective constant domains 331 as previously described $(62,63)$. Heavy-chain Fab constructs contained a HRV 3C-cleavable 332 8xHis tag, while heavy-chain IgG constructs contained HRV 3C-cleavable 8xHis and SBP tags. 333 Transfections and purifications were performed according to the same protocols used for the RBDs 334 and homotrimers.

337 Biolayer interferometry (BLI) experiments were performed using a BLItz instrument (ForteBio). 338 Fabs were immobilized on a FAB2G biosensor (ForteBio), and CoV proteins were the analytes. 339 All proteins were diluted in PBS at room temperature. Titrations were performed to determine 340 binding affinities. Single-hit concentrations at $10 \mu \mathrm{M}$ were performed to get an approximate $K_{D}$, 341 and then subsequent titrations at appropriate concentrations (at least three). A final $K_{D}$ estimate 342 was determined using a global fit model with a 1:1 binding isotherm using vendor-supplied 343 software. 
C57BL/6 mice (Jackson Laboratory) received $20 \mu \mathrm{g}$ of protein adjuvanted with $50 \% \mathrm{w} / \mathrm{v}$ Sigma Adjuvant System in $100 \mu \mathrm{L}$ of inoculum (25). All immunizations were administered through the intraperitoneal route. Mice were primed (day 0) and received boosting immunizations at day 21 and day 42. Serum samples were collected on day 56 for characterization, with flow cytometry occurring between days 56 and 63. In this study, female mice aged 6-10 weeks were used. All

Flow Cytometry

354 Spleens were isolated from mice and single cell suspensions were generated by straining through a $70 \mu \mathrm{m}$ cell strainer. Red blood cells were removed by treating with ACK lysis buffer and washed with PBS. Single cell suspensions were first stained with Aqua Live/Dead amine-reactive dye $(0.025 \mathrm{mg} / \mathrm{mL})$ before applying the following $\mathrm{B}$ and $\mathrm{T}$ cell staining panel using the staining

Streptavidin-conjugated fluorophores were used to label the SBP-tagged proteins as probes for approach described previously $(25,64)$. This included the following mouse-specific antibodies: CD3-BV786 (BioLegend), CD19-BV421 (BioLegend), IgM-BV605 (BioLegend), IgGPerCP/Cy5.5 (BioLegend). flow cytometry. For the cohorts that received the SARS-CoV-2 spike prime followed by either the SARS-CoV-2 RBD homotrimer boost or the RBD homotrimer cocktail boost, the following probes were generated: SARS-CoV-2 RBD-APC/Cy7 (streptavidin-APC/Cy7 from BioLegend), SARSCoV-2 spike-StreptTactin PE (StrepTactin PE from IBA Lifesciences), SARS-CoV-2 $\triangle$ RBM RBD-PE/Cy5.5 (streptavidin-PE/Cy5.5 from BioLegend). The panel for the cohort that received the SARS-CoV-2 spike prime followed by the RBD homotrimer cocktail boost also included 
SARS-CoV RBD-APC (streptavidin-APC from BioLegend) and WIV1-CoV RBD-BV650 (streptavidin-BV650 from BioLegend). For the cohort that received three SARS-CoV-2 spike immunizations, the following probes were generated: SARS-CoV-2 RBD-APC/Cy7 (streptavidinAPC/Cy7 from BioLegend), SARS-CoV-2 spike-StreptTactin PE (StrepTactin PE from IBA Lifesciences), SARS-CoV-2 $\triangle \mathrm{RBM} \quad \mathrm{RBD}-\mathrm{APC} \quad$ (streptavidin-APC from BioLegend). between to achieve a final molar ratio of probe to streptavidin valency of $1: 1$. The final conjugated probe concentration was $0.1 \mu \mathrm{g} / \mathrm{mL}$. Flow cytometry was performed on a BD FACSAria Fusion cytometer (BD Biosciences). Analysis of the resultant FCS files was conducted using FlowJo (version 10).

\section{$\underline{\text { Serum ELISAs }}$}

Serum ELISAs were performed by coating Corning 96-well clear flat bottom high bind microplates with $100 \mu \mathrm{L}$ of protein at $5 \mu \mathrm{g} / \mathrm{mL}$ in PBS. Plates were incubated overnight at $4^{\circ} \mathrm{C}$. Coating solution was removed, and plates were blocked using 1\% BSA in PBS with 1\% Tween for 60 minutes at room temperature. Blocking solution was removed. Sera were diluted 1:40 in PBS, and 5-fold serial dilution was performed. CR3022 $\mathrm{IgG}$ at a starting dilution of $5 \mu \mathrm{g} / \mathrm{mL}$ with 5-fold serial dilution was used as a positive control. $40 \mu \mathrm{L}$ of primary antibody solution was applied to each well. Primary incubation occurred for 90 minutes at room temperature. Plates were then washed three times with PBS-Tween. HRP-conjugated rabbit anti-mouse IgG antibody (Abcam) at a concentration of 1:20,000 in PBS and a volume of $150 \mu \mathrm{L}$ was used as a secondary antibody. Secondary incubation occurred for 60 minutes at room temperature. Plates were then washed three 
times with PBS-Tween. 1xABTS development solution (ThermoFisher) was applied as outlined in the manufacturer's recommendations. Development was stopped after 30 minutes with a 1\% SDS solution. Plates were read at $405 \mathrm{~nm}$ using a SectraMax iD3 plate reader (Molecular Devices).

Competition ELISAs

397 Competition ELISAs were performed using a similar protocol to serum ELISAs. The primary 398 incubation consisted of $40 \mu \mathrm{L}$ of the relevant $\operatorname{IgG}$ at $1 \mu \mathrm{M}$. Incubation occurred at room 399 temperature for 60 minutes. Mouse sera were then spiked in at a final concentration in the linear 400 range of the serum ELISA titration curve (1:800 for the cohort that received three SARS-CoV-2 401 RBD monomer immunizations, 1:12,800 for all other cohorts). Plates were incubated at room 402 temperature for an additional 60 minutes. The primary solution was removed, and plates were washed three times using PBS-Tween. HRP-conjugated goat anti-mouse IgG, human/bovine/horse SP ads antibody (Southern Biotech) was applied at a concentration of 1:4000 and a volume of 150 $\mu \mathrm{L}$ as a secondary antibody. Plates were then incubated, washed, and developed using the same

\section{$\underline{\text { ACE2 Cell Binding Assay }}$}

ACE2 expressing 293T cells (66) (a kind gift from Nir Hacohen and Michael Farzan) were harvested and washed with PBSF. Cells were allocated such that 200,000 cells were labelled for each condition. Cells were incubated with $100 \mu \mathrm{L}$ of $200 \mathrm{nM}$ antigen in PBS for 60 minutes on ice. Following two washes with PBS supplemented with $2 \%$ FBS, cells were incubated with 50 $\mu \mathrm{L}$ of 1:200 streptavidin-PE (Invitrogen) on ice for 30 minutes. Cells were washed twice and 
resuspended in $100 \mu \mathrm{L}$ of PBS supplemented with 2\% FBS. Flow cytometry was performed using

415 a Stratedigm S1000Exi Flow Cytometer. FCS files were analyzed using FlowJo (version 10).

$417 \quad$ Human Serum Samples

418 A total of 24 human serum samples were obtained from a previously characterized cohort (9).

419 All patients had received either 1 or 2 doses of the Pfizer (BNT162b2) or Moderna (mRNA-

420 1273) vaccines. Of the patients who had received both doses, samples were collected a median of

42114 days following the second dose (range: 4 days - 32 days). The median patient age was 51

422 years, with ages ranging from 22-66 years.

$424 \quad \underline{\text { Serum Adsorption }}$

425 SNAP-tagged (61) MERS-CoV RBD, SARS-CoV-2 $\triangle$ RBM RBD with four additional putative 426 glycosylation sites (Figure S5), and SARS-CoV-2 RBD were conjugated to SNAP-Capture Pull427 Down resin (New England BioLabs). For each conjugation, $20 \mu \mathrm{L}$ of settled resin was resuspended 428 in $100 \mu \mathrm{L}$ of protein at $1 \mathrm{mg} / \mathrm{mL}$ per the manufacturer's recommendations. Both MERS-CoV RBD 429 and SARS-CoV-2 RBD conjugation reactions also included $1 \mathrm{mM}$ DTT to improve conjugation 430 efficiency. Wildtype-like reactivity to conformationally specific Fabs (CR3022, S309, and B38 for 431 SARS-CoV-2 RBD; m336 for MERS RBD) in the presence of $1 \mathrm{mM}$ DTT was confirmed prior to 432 conjugation $(15,17,18,67)$. Conjugation occurred with rotation overnight at $4{ }^{\circ} \mathrm{C}$.

434 Following conjugation, the resin was washed 5 times with PBS via resuspension followed by 435 centrifugation and the removal of the supernatant. Sera from the mice in each cohort were pooled 436 and diluted 1:40 in PBS to a total volume of $100 \mu \mathrm{L}$. Sera from human patients were diluted 1:20 
in PBS to a total volume of $100 \mu \mathrm{L}$. Diluted sera were added to the conjugated resin and incubated with rotation overnight at $4^{\circ} \mathrm{C}$. Resin was filtered from the sera following incubation, and the adsorbed sera was used for neutralization assays and ELISAs.

$\underline{\text { Serum Adsorption ELISAs }}$

442 Serum adsorption ELISAs were performed using a similar protocol to the serum ELISAs. For the 443 primary antibody incubation, adsorbed serum solution was diluted 1:2, and subsequent serial 5444 fold dilutions were generated. The remainder of the assay was performed according to the serum $445 \quad$ ELISA procedure.

$447 \quad$ Pseudovirus Neutralization Assay

448 Serum neutralization against SARS-CoV-2, SARS-CoV, and WIV1-CoV was assessed using 449 lentiviral particles pseudotyped with the respective spike proteins as previously described $(8,9)$. 450 Lentiviral particles were produced via transient transfection of $293 \mathrm{~T}$ cells. The titers of viral 451 supernatants were determined via flow cytometry on 293T-ACE2 cells (66) and via the HIV-1 452 p24 $4^{\mathrm{CA}}$ antigen capture assay (Leidos Biomedical Research, Inc.). Assays were performed in 384453 well plates (Grenier) using a Fluent Automated Workstation (Tecan). For mouse sera, samples $454 \quad$ were initially diluted 1:9, with subsequent serial 3 -fold dilutions. For human and mouse sera that 455 had previously been adsorbed, the adsorbed sample was used at an initial 1:3 dilution, and serial 4563 -fold dilutions were performed. Serum sample volume in each well was $20 \mu \mathrm{L}$, and $20 \mu \mathrm{L}$ of 457 pseudovirus containing 125 infectious units was added. The combination was incubated for 60 458 minutes at room temperature. Afterwards, 10,000 293T-ACE2 cells (66) in $20 \mu \mathrm{L}$ of media 
459 containing $15 \mu \mathrm{g} / \mathrm{mL}$ polybrene was added. The plates were then incubated at $37^{\circ} \mathrm{C}$ for $60-72$ 460 hours.

462 A previously described assay buffer was used to lyse the cells (68). A Spectramax L luminometer 463 (Molecular Devices) was used to quantify luciferase expression. Percent neutralization at each 464 serum concentration was determined by subtracting background luminescence from cells only 465 sample wells, then dividing by luminescence of wells with only virus and cells. GraphPad Prism 466 was used to fit nonlinear regressions to the data, which allowed $\mathrm{IC}_{50}$ values to be calculated using 467 the interpolated $50 \%$ inhibitory concentration. $\mathrm{IC}_{50}$ values were calculated for all samples with a 468 neutralization value of at least $80 \%$ at the highest serum concentration.

\section{$470 \quad$ Phylogenetic Trees}

471 Prior to generating phylogenetic trees, alignments were generated via ClustalOmega. Phylogenetic 472 trees were then generated using those alignments as inputs into a neighbor-joining algorithm via 473 ClustalW2. All settings were left as default.

$475 \quad$ Statistical Analysis

476 Statistical analyses and curve fitting were performed using GraphPad Prism (version 9). To 477 compare two populations of continuous variables without evidence of conforming to a normal 478 distribution, the non-parametric two-tailed Mann-Whitney U test was used. To compare multiple 479 populations meeting this description, the Kruskal-Wallis test was used with post hoc analysis using 480 Dunn's multiple comparison testing. The ratio paired t-test was used to compare two populations 
bioRxiv preprint doi: https://doi.org/10.1101/2020.12.07.415216; this version posted June 29, 2021. The copyright holder for this preprint (which was not certified by peer review) is the author/funder. All rights reserved. No reuse allowed without permission.

481 with evidence of normality. P values were corrected for multiple comparisons, and a $\mathrm{p}$ value $<$ $482 \quad 0.05$ was considered significant.

483

484 


\section{References:}

1. Amanat F, Krammer F. SARS-CoV-2 Vaccines: Status Report. Immunity. 2020;52(4):583-9.

2. Anthony SJ, Johnson CK, Greig DJ, Kramer S, Che X, Wells H, et al. Global patterns in coronavirus diversity. Virus Evol. 2017;3(1):vex012.

3. Boni MF, Lemey P, Jiang X, Lam TT, Perry BW, Castoe TA, et al. Evolutionary origins of the SARS-CoV-2 sarbecovirus lineage responsible for the COVID-19 pandemic. Nat Microbiol. 2020. Emerging Zoonotic Diseases. Keusch GT PM, Gonzalez MC, et al., editor. Washington (DC): National Academies Press (US); 2009. 5. Jiang S, Hillyer C, Du L. Neutralizing Antibodies against SARS-CoV-2 and Other Human Coronaviruses. Trends Immunol. 2020;41(5):355-9.

6. Du L, He Y, Zhou Y, Liu S, Zheng BJ, Jiang S. The spike protein of SARS-CoV--a target for vaccine and therapeutic development. Nat Rev Microbiol. 2009;7(3):226-36. 7. Yang W, Kandula S, Huynh M, Greene SK, Van Wye G, Li W, et al. Estimating the infection-fatality risk of SARS-CoV-2 in New York City during the spring 2020 pandemic wave: a model-based analysis. Lancet Infect Dis. 2020.

8. Garcia-Beltran WF, Lam EC, Astudillo MG, Yang D, Miller TE, Feldman J, et al. COVID-19-neutralizing antibodies predict disease severity and survival. Cell. 2021;184(2):47688 e11.

9. Garcia-Beltran WF, Lam EC, St Denis K, Nitido AD, Garcia ZH, Hauser BM, et al. Multiple SARS-CoV-2 variants escape neutralization by vaccine-induced humoral immunity. Cell. 2021.

10. Knipe DM, Howley PM. Fields virology. 6th ed. Philadelphia, PA: Wolters Kluwer/Lippincott Williams \& Wilkins Health; 2013. 2 volumes p.

11. Wec AZ, Wrapp D, Herbert AS, Maurer DP, Haslwanter D, Sakharkar M, et al. Broad neutralization of SARS-related viruses by human monoclonal antibodies. Science. 2020.

12. Yuan M, Liu H, Wu NC, Lee CD, Zhu X, Zhao F, et al. Structural basis of a shared antibody response to SARS-CoV-2. Science. 2020.

13. Zost SJ, Gilchuk P, Case JB, Binshtein E, Chen RE, Nkolola JP, et al. Potently neutralizing and protective human antibodies against SARS-CoV-2. Nature. 2020.

14. Rogers TF, Zhao F, Huang D, Beutler N, Burns A, He WT, et al. Isolation of potent SARS-CoV-2 neutralizing antibodies and protection from disease in a small animal model. Science. 2020.

15. Wu Y, Wang F, Shen C, Peng W, Li D, Zhao C, et al. A noncompeting pair of human neutralizing antibodies block COVID-19 virus binding to its receptor ACE2. Science. 2020;368(6496):1274-8.

16. Du L, Yang Y, Zhou Y, Lu L, Li F, Jiang S. MERS-CoV spike protein: a key target for antivirals. Expert Opin Ther Targets. 2017;21(2):131-43.

17. Yuan M, Wu NC, Zhu X, Lee CD, So RTY, Lv H, et al. A highly conserved cryptic epitope in the receptor binding domains of SARS-CoV-2 and SARS-CoV. Science.

2020;368(6491):630-3. 
18. Pinto D, Park YJ, Beltramello M, Walls AC, Tortorici MA, Bianchi S, et al. Crossneutralization of SARS-CoV-2 by a human monoclonal SARS-CoV antibody. Nature. 2020;583(7815):290-5.

19. Dai L, Zheng T, Xu K, Han Y, Xu L, Huang E, et al. A Universal Design of

Betacoronavirus Vaccines against COVID-19, MERS, and SARS. Cell. 2020;182(3):722-33 e11. 20. Menachery VD, Yount BL, Jr., Sims AC, Debbink K, Agnihothram SS, Gralinski LE, et al. SARS-like WIV1-CoV poised for human emergence. Proc Natl Acad Sci U S A. 2016;113(11):3048-53.

21. Fonville JM, Wilks SH, James SL, Fox A, Ventresca M, Aban M, et al. Antibody landscapes after influenza virus infection or vaccination. Science. 2014;346(6212):996-1000.

22. Sliepen K, van Montfort T, Melchers M, Isik G, Sanders RW. Immunosilencing a highly immunogenic protein trimerization domain. J Biol Chem. 2015;290(12):7436-42.

23. Pallesen J, Wang N, Corbett KS, Wrapp D, Kirchdoerfer RN, Turner HL, et al. Immunogenicity and structures of a rationally designed prefusion MERS-CoV spike antigen. Proc Natl Acad Sci U S A. 2017;114(35):E7348-E57.

24. Corbett KS, Edwards DK, Leist SR, Abiona OM, Boyoglu-Barnum S, Gillespie RA, et al. SARS-CoV-2 mRNA vaccine design enabled by prototype pathogen preparedness. Nature. 2020;586(7830):567-71.

25. Sangesland M, Ronsard L, Kazer SW, Bals J, Boyoglu-Barnum S, Yousif AS, et al. Germline-Encoded Affinity for Cognate Antigen Enables Vaccine Amplification of a Human Broadly Neutralizing Response against Influenza Virus. Immunity. 2019;51(4):735-49 e8.

26. Lan J, Ge J, Yu J, Shan S, Zhou H, Fan S, et al. Structure of the SARS-CoV-2 spike receptor-binding domain bound to the ACE2 receptor. Nature. 2020;581(7807):215-20.

27. Barnes CO, Jette CA, Abernathy ME, Dam KA, Esswein SR, Gristick HB, et al. SARSCoV-2 neutralizing antibody structures inform therapeutic strategies. Nature. 2020.

28. Menachery VD, Yount BL, Jr., Debbink K, Agnihothram S, Gralinski LE, Plante JA, et al. A SARS-like cluster of circulating bat coronaviruses shows potential for human emergence. Nat Med. 2015;21(12):1508-13.

29. Shang J, Ye G, Shi K, Wan Y, Luo C, Aihara H, et al. Structural basis of receptor recognition by SARS-CoV-2. Nature. 2020;581(7807):221-4.

30. Cohen AA, Gnanapragasam PNP, Lee YE, Hoffman PR, Ou S, Kakutani LM, et al. Mosaic nanoparticles elicit cross-reactive immune responses to zoonotic coronaviruses in mice. Science. 2021;371(6530):735-41.

31. Abu-Raddad LJ, Chemaitelly H, Butt AA, National Study Group for C-V. Effectiveness of the BNT162b2 Covid-19 Vaccine against the B.1.1.7 and B.1.351 Variants. N Engl J Med. 2021.

32. Edara VV, Lai L, Sahoo MK, Floyd K, Sibai M, Solis D, et al. Infection and vaccineinduced neutralizing antibody responses to the SARS-CoV-2 B.1.617.1 variant. bioRxiv. 2021. 33. Wu K, Werner AP, Koch M, Choi A, Narayanan E, Stewart-Jones GBE, et al. Serum Neutralizing Activity Elicited by mRNA-1273 Vaccine. N Engl J Med. 2021;384(15):1468-70. 34. Collier DA, De Marco A, Ferreira I, Meng B, Datir RP, Walls AC, et al. Sensitivity of SARS-CoV-2 B.1.1.7 to mRNA vaccine-elicited antibodies. Nature. 2021;593(7857):136-41. 35. Shapiro J, Dean NE, Madewell ZJ, Yang Y, Halloran ME, Longini I. Efficacy Estimates for Various COVID-19 Vaccines: What we Know from the Literature and Reports. medRxiv. 2021. 
36. Martinez DR, Schaefer A, Leist SR, De la Cruz G, West A, Atochina-Vasserman EN, et al. Chimeric spike mRNA vaccines protect against sarbecovirus challenge in mice. Science. 2021.

37. Walls AC, Fiala B, Schafer A, Wrenn S, Pham MN, Murphy M, et al. Elicitation of Potent Neutralizing Antibody Responses by Designed Protein Nanoparticle Vaccines for SARSCoV-2. Cell. 2020;183(5):1367-82 e17.

38. Wang W, Huang B, Zhu Y, Tan W, Zhu M. Ferritin nanoparticle-based SARS-CoV-2 RBD vaccine induces a persistent antibody response and long-term memory in mice. Cell Mol Immunol. 2021;18(3):749-51.

39. Kang YF, Sun C, Zhuang Z, Yuan RY, Zheng Q, Li JP, et al. Rapid Development of SARS-CoV-2 Spike Protein Receptor-Binding Domain Self-Assembled Nanoparticle Vaccine Candidates. ACS Nano. 2021;15(2):2738-52.

40. Li H, Guo L, Zheng H, Li J, Zhao X, Li J, et al. Self-Assembling Nanoparticle Vaccines Displaying the Receptor Binding Domain of SARS-CoV-2 Elicit Robust Protective Immune Responses in Rhesus Monkeys. Bioconjug Chem. 2021;32(5):1034-46.

41. Liu Z, Xu W, Xia S, Gu C, Wang X, Wang Q, et al. RBD-Fc-based COVID-19 vaccine candidate induces highly potent SARS-CoV-2 neutralizing antibody response. Signal Transduct Target Ther. 2020;5(1):282.

42. Yang J, Wang W, Chen Z, Lu S, Yang F, Bi Z, et al. A vaccine targeting the RBD of the S protein of SARS-CoV-2 induces protective immunity. Nature. 2020;586(7830):572-7.

43. Ma X, Zou F, Yu F, Li R, Yuan Y, Zhang Y, et al. Nanoparticle Vaccines Based on the Receptor Binding Domain (RBD) and Heptad Repeat (HR) of SARS-CoV-2 Elicit Robust Protective Immune Responses. Immunity. 2020;53(6):1315-30 e9.

44. Huang WC, Zhou S, He X, Chiem K, Mabrouk MT, Nissly RH, et al. SARS-CoV-2 RBD Neutralizing Antibody Induction is Enhanced by Particulate Vaccination. Adv Mater. 2020;32(50):e2005637.

45. Chen RE, Zhang X, Case JB, Winkler ES, Liu Y, VanBlargan LA, et al. Resistance of SARS-CoV-2 variants to neutralization by monoclonal and serum-derived polyclonal antibodies. Nat Med. 2021;27(4):717-26.

46. Suryadevara N, Shrihari S, Gilchuk P, VanBlargan LA, Binshtein E, Zost SJ, et al. Neutralizing and protective human monoclonal antibodies recognizing the $\mathrm{N}$-terminal domain of the SARS-CoV-2 spike protein. Cell. 2021;184(9):2316-31 e15.

47. Chi X, Yan R, Zhang J, Zhang G, Zhang Y, Hao M, et al. A neutralizing human antibody binds to the N-terminal domain of the Spike protein of SARS-CoV-2. Science. 2020;369(6504):650-5.

48. Hansen J, Baum A, Pascal KE, Russo V, Giordano S, Wloga E, et al. Studies in humanized mice and convalescent humans yield a SARS-CoV-2 antibody cocktail. Science. 2020;369(6506):1010-4.

49. Rappazzo CG, Tse LV, Kaku CI, Wrapp D, Sakharkar M, Huang D, et al. Broad and potent activity against SARS-like viruses by an engineered human monoclonal antibody. Science. 2021;371(6531):823-9. domain than do those from SARS-CoV-2 infection. Sci Transl Med. 2021. 
51. Jones BE, Brown-Augsburger PL, Corbett KS, Westendorf K, Davies J, Cujec TP, et al. The neutralizing antibody, LY-CoV555, protects against SARS-CoV-2 infection in nonhuman primates. Sci Transl Med. 2021;13(593).

52. Wu LP, Wang NC, Chang YH, Tian XY, Na DY, Zhang LY, et al. Duration of antibody responses after severe acute respiratory syndrome. Emerg Infect Dis. 2007;13(10):1562-4.

53. Drosten C, Meyer B, Muller MA, Corman VM, Al-Masri M, Hossain R, et al. Transmission of MERS-coronavirus in household contacts. N Engl J Med. 2014;371(9):828-35. 54. Sariol A, Perlman S. Lessons for COVID-19 Immunity from Other Coronavirus Infections. Immunity. 2020;53(2):248-63.

55. Schmidt OW, Allan ID, Cooney MK, Foy HM, Fox JP. Rises in titers of antibody to human coronaviruses OC43 and 229E in Seattle families during 1975-1979. Am J Epidemiol. 1986;123(5):862-8.

56. Monto AS, Lim SK. The Tecumseh study of respiratory illness. VI. Frequency of and relationship between outbreaks of coronavirus infection. J Infect Dis. 1974;129(3):271-6.

57. Hendley JO, Fishburne HB, Gwaltney JM, Jr. Coronavirus infections in working adults. Eight-year study with 229 E and OC 43. Am Rev Respir Dis. 1972;105(5):805-11.

58. Callow KA, Parry HF, Sergeant M, Tyrrell DA. The time course of the immune response to experimental coronavirus infection of man. Epidemiol Infect. 1990;105(2):435-46.

59. Gorman MJ, Patel N, Guebre-Xabier M, Zhu A, Atyeo C, Pullen KM, et al. Collaboration between the Fab and Fc contribute to maximal protection against SARS-CoV-2 in nonhuman primates following NVX-CoV2373 subunit vaccine with Matrix-M vaccination. bioRxiv. 2021.

60. Weisblum Y, Schmidt F, Zhang F, DaSilva J, Poston D, Lorenzi JC, et al. Escape from neutralizing antibodies by SARS-CoV-2 spike protein variants. Elife. 2020;9.

61. Keppler A, Gendreizig S, Gronemeyer T, Pick H, Vogel H, Johnsson K. A general method for the covalent labeling of fusion proteins with small molecules in vivo. Nat Biotechnol. 2003;21(1):86-9.

62. Schmidt AG, Do KT, McCarthy KR, Kepler TB, Liao HX, Moody MA, et al. Immunogenic Stimulus for Germline Precursors of Antibodies that Engage the Influenza Hemagglutinin Receptor-Binding Site. Cell Rep. 2015;13(12):2842-50.

63. Schmidt AG, Therkelsen MD, Stewart S, Kepler TB, Liao HX, Moody MA, et al. Viral receptor-binding site antibodies with diverse germline origins. Cell. 2015;161(5):1026-34.

64. Weaver GC, Villar RF, Kanekiyo M, Nabel GJ, Mascola JR, Lingwood D. In vitro reconstitution of $\mathrm{B}$ cell receptor-antigen interactions to evaluate potential vaccine candidates. Nat Protoc. 2016;11(2):193-213.

65. Kaneko N, Kuo HH, Boucau J, Farmer JR, Allard-Chamard H, Mahajan VS, et al. Loss of Bcl-6-Expressing T Follicular Helper Cells and Germinal Centers in COVID-19. Cell. 2020;183(1):143-57 e13.

66. Moore MJ, Dorfman T, Li W, Wong SK, Li Y, Kuhn JH, et al. Retroviruses pseudotyped with the severe acute respiratory syndrome coronavirus spike protein efficiently infect cells expressing angiotensin-converting enzyme 2. J Virol. 2004;78(19):10628-35.

67. Ying T, Du L, Ju TW, Prabakaran P, Lau CC, Lu L, et al. Exceptionally potent neutralization of Middle East respiratory syndrome coronavirus by human monoclonal antibodies. J Virol. 2014;88(14):7796-805.

68. Siebring-van Olst E, Vermeulen C, de Menezes RX, Howell M, Smit EF, van Beusechem VW. Affordable luciferase reporter assay for cell-based high-throughput screening. J Biomol Screen. 2013;18(4):453-61. 
Acknowledgments: We thank members of the Schmidt and Lingwood Laboratories for helpful 665 discussions. We thank Dr. Jason McLellan from University of Texas, Austin for the spike plasmid. 666 We thank Nir Hacohen and Michael Farzan for the kind gift of the ACE2 expressing 293T cells to 667 ABB.; Funding: We acknowledge funding from NIH R01s AI146779 (AGS), AI124378, 668 AI137057 and AI153098 (DL), and a Massachusetts Consortium on Pathogenesis Readiness 669 (MassCPR) grant (AGS); training grants: NIGMS T32 GM007753 (BMH and TMC); T32 AI007245 (JF); F31 A1138368 (MS). A.B.B. is supported by the National Institutes for Drug Abuse (NIDA) Avenir New Innovator Award DP2DA040254, the MGH Transformative Scholars 672 Program as well as funding from the Charles H. Hood Foundation. This independent research was 673 supported by the Gilead Sciences Research Scholars Program in HIV.; Author contributions: 674 Conceptualization, BMH, MS, JF, TMC, DL, AGS; Methodology, BMH, ECL, TMC, ABB, DL, 675 AGS; Investigation, BMH, MS, ECL, KSD, JF, ASY, TK; Writing - Original Draft, BMH and 676 AGS; Writing - Review and Editing, all authors; Funding Acquisition, ABB, DL, AGS; Supervision, ABB, DL, AGS.; Competing interests: Authors declare no competing interests.; and materials. 


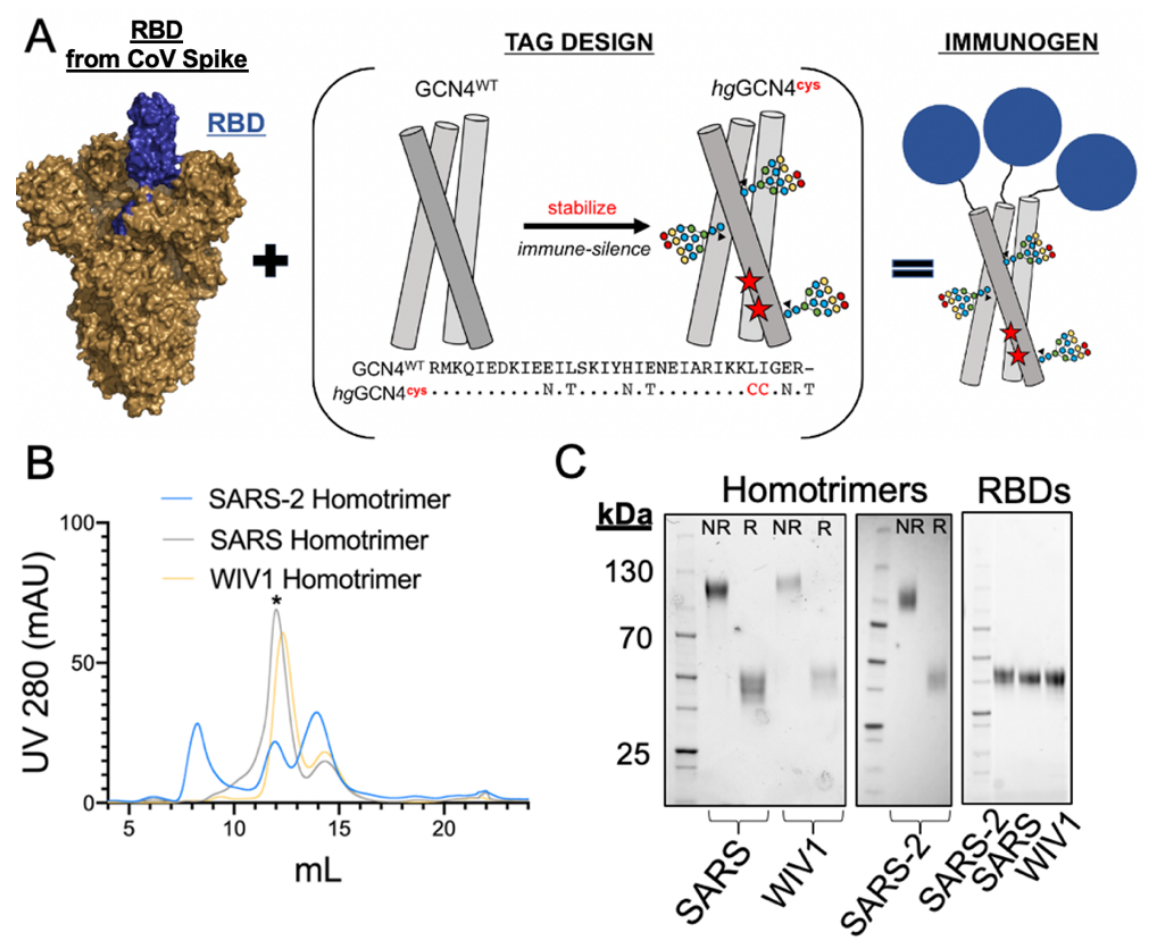

Fig. 1. Immunogen design, expression, and purification. (A) Design schematic for generating RBD homotrimers appended onto a cystine-stabilized (red stars) hyperglycosylated GCN4 tag. (PDB: 6VSB) (B) Representative size exclusion trace with $\left(^{*}\right)$ marking the homotrimeric construct. Fractions in this peak were pooled and used for immunizations. (C) SDS-PAGE analysis of purified homotrimers following removal of the affinity purification tags under non-reducing (NR) and reducing $(\mathrm{R})$ conditions. The engineered disulfide bond at the C-terminus of the hyperglycosylated GCN4 tag separated under reducing conditions. Panel includes monomeric 690 


\begin{tabular}{|c|c|c|c|}
\hline Cohort ID & Day 0 & Day 21 & Day 42 \\
\hline Spike & SARS-2 Spike & SARS-2 Spike & SARS-2 Spike \\
\hline RBD & SARS-2 RBD & SARS-2 RBD & SARS-2 RBD \\
\hline Homotrimer & SARS-2 Spike & SARS-2 Homotrimer & SARS-2 Homotrimer \\
\hline Cocktail & SARS-2 Spike & $\begin{array}{c}\text { RBD Homotrimer } \\
\text { Cocktail }\end{array}$ & $\begin{array}{c}\text { RBD Homotrimer } \\
\text { Cocktail }\end{array}$ \\
\hline
\end{tabular}
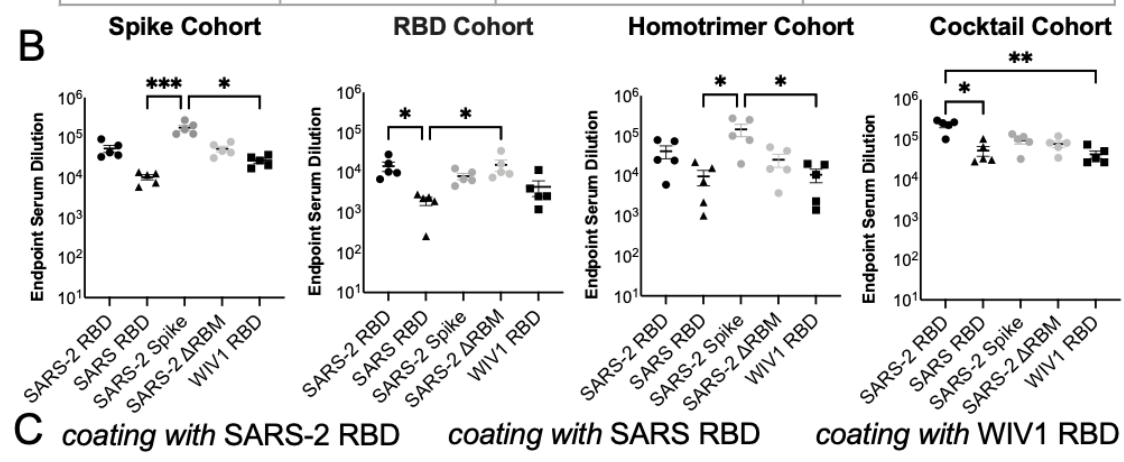

coating with SARS RBD

coating with WIV1 RBD
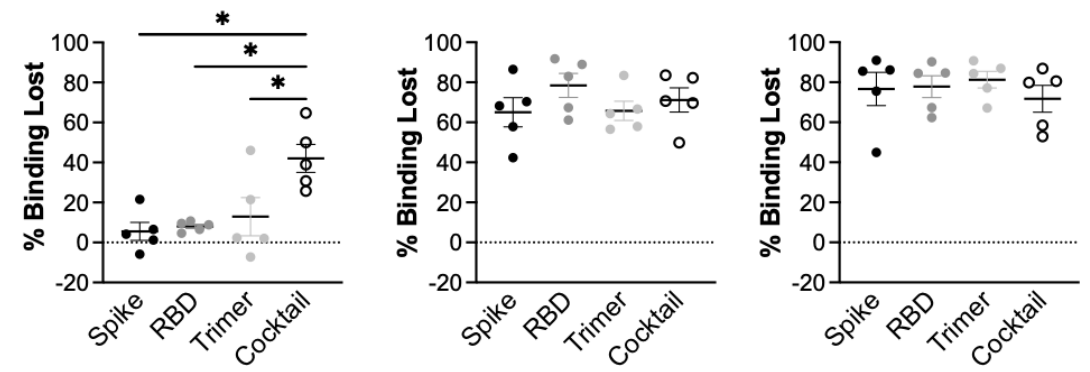

Fig. 2. Serum response to immunization regimens. (A) Four immunization cohorts were used for this study ( $\mathrm{n}=5$ mice, per cohort). (B) Serum was assayed in ELISA at day 56 with different coronavirus antigens. (C) Percent of binding lost in competition ELISAs using S309 and CR3022 vs. no IgG and SARS-CoV-2, SARS-CoV, and WIV1-CoV RBDs as coating antigens. Statistical corrected for multiple comparisons $(*=\mathrm{p}<0.05, * *=\mathrm{p}<0.01$, *** $=\mathrm{p}<0.001)$; ns $=$ not significant. (Related to Fig. S2) 


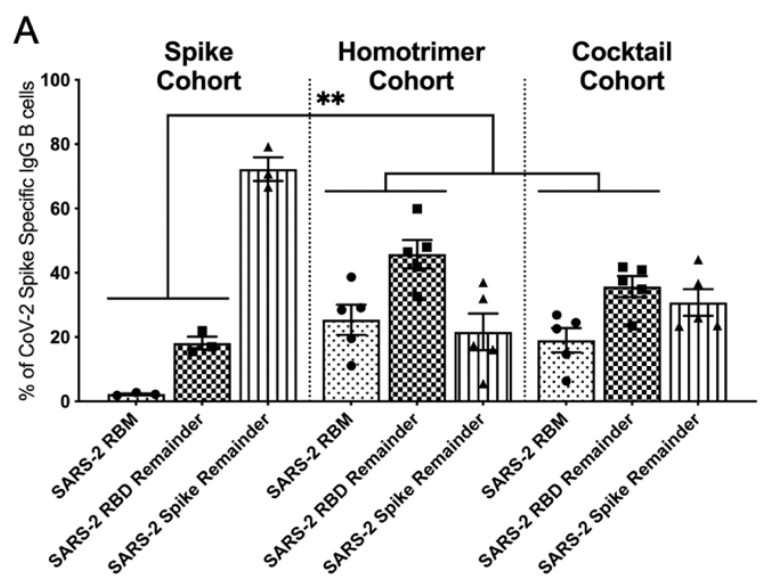

B

Cocktail Cohort

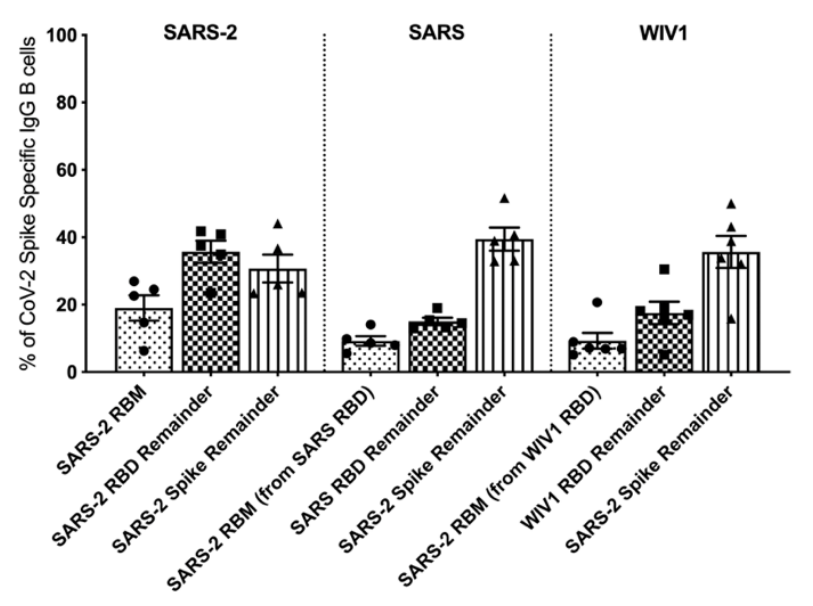

Fig. 3. SARS-CoV-2-specific IgG B cells expanded following immunization. (A) Spikedirected responses were binned into RBM, RBD remainder (excluding RBM epitopes), and spike remainder (excluding $\mathrm{RBD}$ and $\mathrm{RBM}$ epitopes) populations using relevant probes in flow cytometry. Data is shown as a percentage of total spike-specific IgG B-cells. We evaluated the distribution of SARS-CoV-2 spike-directed responses and found evidence of focusing towards non-spike RBD epitopes in the cohorts boosted with the SARS-CoV-2 RBD homotrimer and the RBD homotrimer cocktail versus the cohort that received three SARS-CoV-2 spike immunizations ( $\mathrm{p}=0.0070$ via Mann-Whitney U test) (A). (B) The cocktail cohort was additionally assayed for SARS-CoV or WIV1-CoV RBD reactivity, as described in (A). (Related to Fig. S3) 

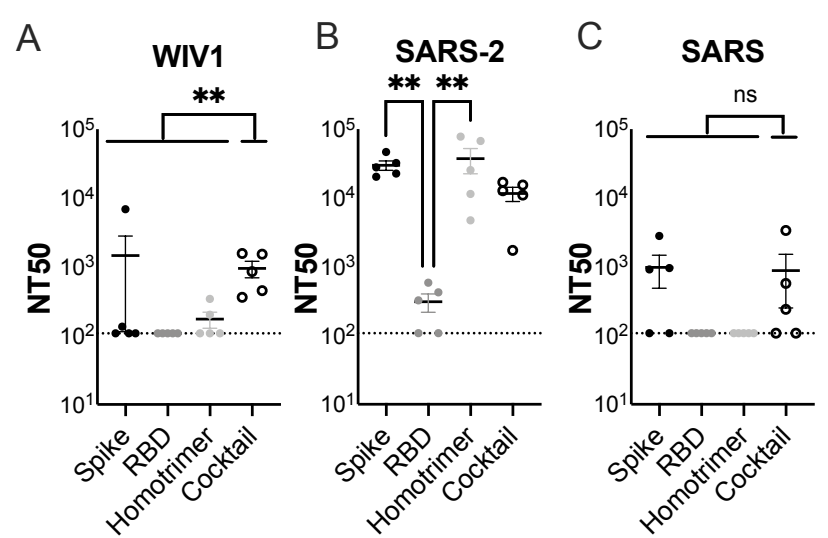

\section{D \\ RBD}
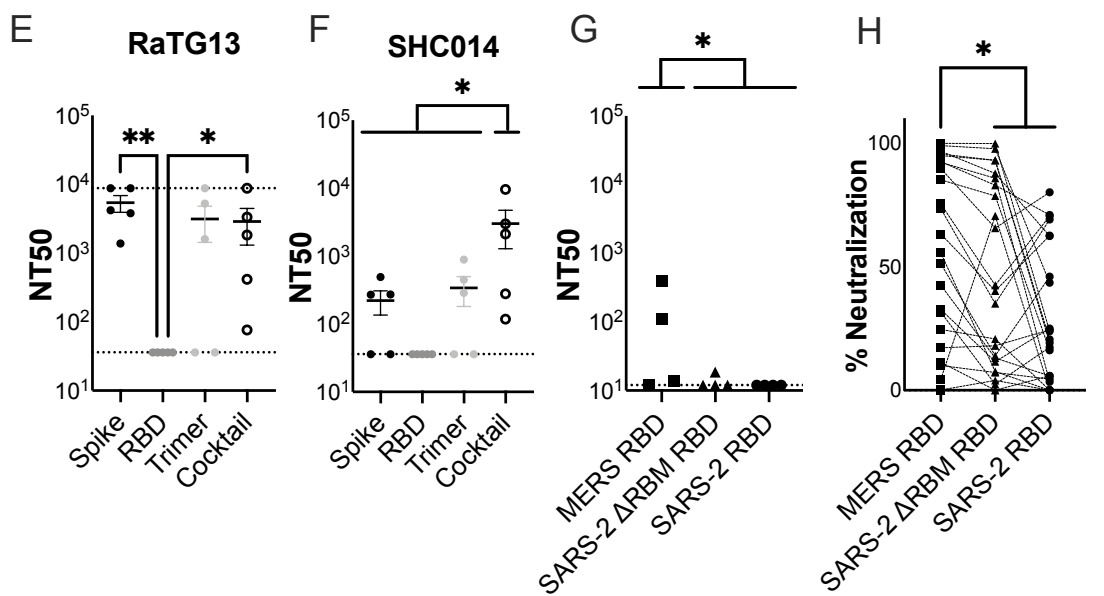

Fig. 4. Pan-sarbecovirus serum neutralization using pseudoviruses. Day 56 serum from each cohort was assayed for neutralization against (A) WIV1-CoV,

(B) SARS-CoV-2,

(C) SARS-CoV, (E) RaTG13-CoV, and (F) SHC014-CoV pseudoviruses. For the SARS-CoV-2 (B) and RaTG13$\mathrm{CoV}$ (E) pseudoviruses, statistical significance was determined using Kruskal-Wallis test with post-hoc analysis using Dunn's test; $*=\mathrm{p}<0.05, * *=\mathrm{p}<0.01$. There was a significant difference in WIV1-CoV neutralization (Mann-Whitney $\mathrm{U}$ test; $\mathrm{p}=0.0012$ ) (A) and SHC014-CoV neutralization (Mann-Whitney $U$ test; $p=0.0145)(\mathbf{F})$, but there was no significant difference in SARS-CoV neutralization between the control cohorts and the RBD homotrimer cocktail boost cohort (Mann-Whitney U test; $\mathrm{p}=0.2370)(\mathbf{C})$.

(D) Neighbor-joining phylogenetic trees were generated to describe the phylogenetic relationships between the RBDs and spikes of the coronaviruses used in this neutralization panel. Branch lengths are displayed in parentheses. $(\mathbf{G})$ 
730 adsorbed with MERS-CoV RBD had significantly greater neutralization than those adsorbed with 731 SARS-CoV-2 $\triangle$ RBM RBD and SARS-CoV-2 RBD (Mann-Whitney U test; $p=0.0343$ ). (H) Same 732 assay in $(\mathbf{G})$, except on immune sera from humans who received the Pfizer (BNT162b2) or 733 Moderna (mRNA-1273) vaccine (Mann-Whitney U test; $p=0.0253)$. In $(\mathbf{H})$, percent neutralization 734 was assessed at a 1:3 dilution of the adsorbed sample. Negative percent neutralization values in $735 \quad$ (H) were set to zero to facilitate analysis. (Related to Fig. S4) 